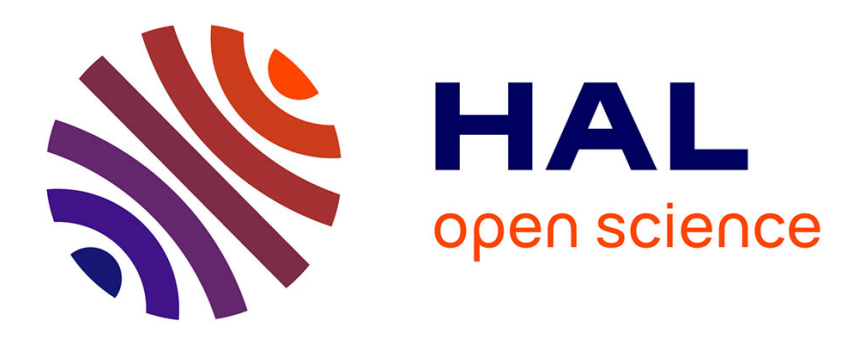

\title{
Coupling between nematic lyomesophases and ferrofluids
}

L. Liebert, A. Martinet

\section{- To cite this version:}

L. Liebert, A. Martinet. Coupling between nematic lyomesophases and ferrofluids. Journal de Physique Lettres, 1979, 40 (15), pp.363-368. 10.1051/jphyslet:019790040015036300 . jpa-00231645

\section{HAL Id: jpa-00231645 https://hal.science/jpa-00231645}

Submitted on 1 Jan 1979

HAL is a multi-disciplinary open access archive for the deposit and dissemination of scientific research documents, whether they are published or not. The documents may come from teaching and research institutions in France or abroad, or from public or private research centers.
L'archive ouverte pluridisciplinaire HAL, est destinée au dépôt et à la diffusion de documents scientifiques de niveau recherche, publiés ou non, émanant des établissements d'enseignement et de recherche français ou étrangers, des laboratoires publics ou privés. 


\title{
Coupling between nematic lyomesophases and ferrofluids
}

\author{
L. Liebert and A. Martinet \\ Laboratoire de Physique des Solides, Université Paris-Sud, 91405 Orsay, France
}

(Reçu le 6 avril 1979, accepté le 8 juin 1979)

\begin{abstract}
Résumé. - Une compatibilité entre colloïde magnétique et une phase nématique lyotrope a été trouvée. Il en résulte une ferronématolyotrope stable. Un couplage entre les grains magnétiques du colloïde et les micelles de la phase lyotrope a été mis en évidence. On obtient un gain par un facteur $10^{3}$ dans les valeurs du champ magnétique nécessaires pour orienter un échantillon, par rapport au cas sans colloïde magnétique.
\end{abstract}

Abstract. - We have been able to disperse a magnetic colloid in a nematic lyomesophase resulting in a stable ferronematolyotrope. A coupling between the magnetic grains and the micelles of the lyomesophase has been observed. The value of the magnetic field necessary to orient a sample is reduced by a factor $10^{3}$ in comparison with the pure nematic lyomesophase.

1. Introduction. - Lawson and Flautt [1] and Rosevear [2] first reported the existence and the properties of nematic lyomesophases. They are made of mixtures of amphiphilic compounds and water. Later, N.M.R. studies showed that these mesophases can have positive or negative $[3,4]$ diamagnetic aniso-

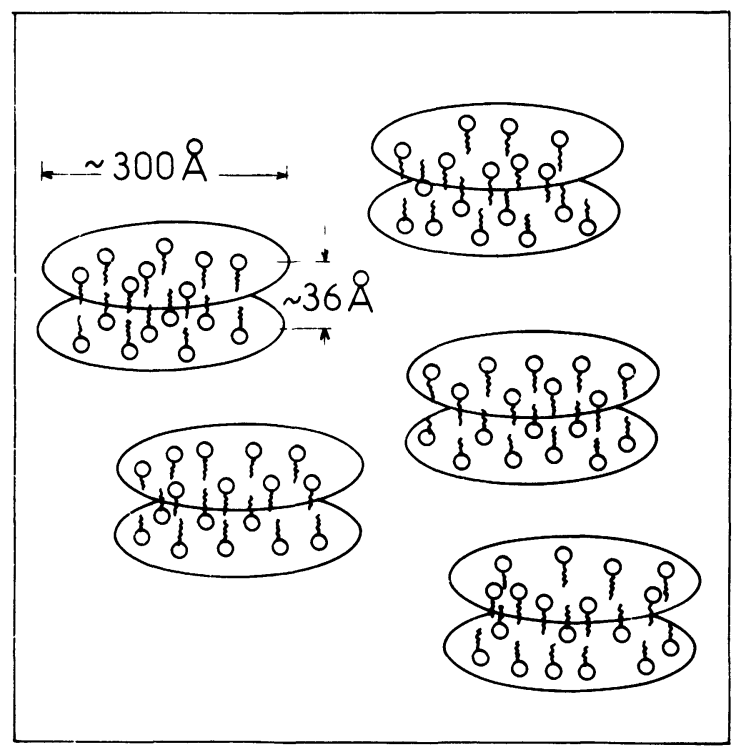

Fig. 1. - Schematic representation of the structure of the type II nematic lyomesophase. The salts with alcohol form disk-shaped micelles immersed in water. This situation corresponds to a sample which has been oriented by applying successively a strong magnetic field in two directions of the horizontal plane. tropies. The phase with positive diamagnetic anisotropy (type I) consists of cylindrical micelles and the other phase (type II) of bilayer micelles. In this paper we only consider the type II because it has been the most studied and it seems that its structure is the less controversial $[3,5]$. Figure 1 shows the generally accepted structure for the type II phase.

It is reasonable to think that the presence of water in these phases would lead them to be compatible with some magnetic colloid. The water between the micelles provides space for the magnetic grains without strongly perturbing the order of the nematic lyomesophase. These materials should have remarkable magnetic properties as theoretically predicted by Brochard and de Gennes [6] for thermotropic liquid crystals. In fact, we have prepared some mixtures of type IIferrofluids with a weight percentage of $\lesssim 1 \%$, resulting in a well dispersed ferronematic lyomesophase. The samples are stable over long periods (many months). We compare the effect of a magnetic field on the orientation of type II samples with and without ferrofluid.

\section{Sample preparation and experimental set-up. -} We have prepared a ternary phase with the following composition in weight : $35.9 \%$ of sodium decylsulphate, $7.2 \%$ of decanol and $56.9 \%$ of $\mathrm{D}_{2} \mathrm{O}$ as described by Reeves et al. [3]. This mixture is type II at room temperature (about $20^{\circ} \mathrm{C}$ ) and changes slowly to another phase (perhaps of type I) at $28^{\circ} \mathrm{C}$. The 
N.M.R. study [7] of this mixture confirms the experimental data given by Reeves. The ferrofluid ( ${ }^{1}$ ) (magnetic colloid of magnetite $\left(\mathrm{Fe}_{3} \mathrm{O}_{4}\right)$ grains suspended in water) is added and the components are mixed by stirring to form a stable blend at room temperature. Spontaneous dispersion does occur. The presence of this amount of ferrofluid does not disturb the optical properties of the type II. We have examined microscopically the sample which shows the same texture with typical defects of the type II. It is well known $[8,9]$ that this water-base ferrofluid tends to form chains. The largest clusters are removed by centrifugation $\left(10 \mathrm{~min}\right.$ at $\left.10^{4} \mathrm{~g}\right)$. The smallest clusters remain. Thus the magnetic material has an acicular (needle) effective shape which is necessary for these experiments. Brochard and de Gennes [6] have shown that the coupling between the grains and the liquid crystal matrix is essentially of mechanical origin : the liquid crystal orients along the direction imposed by the magnetic units to minimize the distortion energy.

The magnetization curve of the resulting sample gives the concentration, the average size of the grains and information on the size distribution [10]. A leastsquare fit of the magnetization curve to a Langevin function and a convolution with a Gaussian size distribution law, gives typically :

$\begin{array}{ll}\text { mean diameter } & : 154 \AA \\ \text { standard deviation } & : 94 \AA \\ \text { number of grains per } \mathrm{cm}^{3} & : 1.9 \times 10^{14} \\ \text { magnetization at saturation } & : 4 \pi M=2.20 \mathrm{G} / \mathrm{cm}^{3} .\end{array}$

The sample is put into a flat capillary with a rectangular cross section which has been prepared by tightly cementing a microscope slide, a polyethylene spacer and a glass cover. The glass cover $\left({ }^{2}\right)$ is provided

( $\left.{ }^{1}\right)$ The ferrofluid was purchased from « Ferrofluidics Company ", reference : water base AO1 $200 \mathrm{G}$.

$\left({ }^{2}\right)$ This material was purchased from "Hellma Company", reference : top cover of 136 OS tank. with two small tubes $(1 \mathrm{~cm}$ long, $2 \times 5 \mathrm{~mm}$ in diameter) for injecting the material. To prevent evaporation, the two glass tubes were connected with a flexible rubber tube. The internal size of the capillary was typically $3 \mathrm{~cm}$ long, $200 \mu$ wide and $150 \mu$ thick.

In the case of type II without ferrofluid, the sample is then put into the gap of an electromagnet ( $H$ maximum $\simeq 16 \mathrm{kG}$ ) which also contains an optical microscope. In the case of type II with ferrofluid, a magnetic field ( $H$ maximum $\simeq 20 \mathrm{G}$ ) can be applied in any direction by using a set of three pairs of Helmoltz coils.

3. Experimental results. - The sample is oriented by the flow created during the filling of the capillary. No measurements are made until (the capillary is full and) the flow has stopped. Residual flow may be as low as $0.1 \mu / \mathrm{s}$. Under the microscope and with crossed polarizers the microscope reveals black lines parallel to the capillary axis. These correspond to the sites where the optical axis is parallel or perpendicular to the vibrating directions of the polarizers. The two possibilities are distinguished by looking at the response to a magnetic field (see below).

We have determined the envelope of the major axis of the disk projection over the plane of observation (i.e. the profile of the micelle orientation across the capillary), by measuring the position of the black lines versus the angle $\theta$ between the polar vibration and the capillary axis (see plate I). For better precision, the capillary is held steady, the crossed polars are rotated and the line position is determined through a micrometric ocular with shiftable index. As optically spurious reflexions occur at the boundaries of the capillary, the width of the channel cannot be determined to better than $\pm 3 \%$. The profile is measured with and without an applied magnetic field.

3.1 TYPE II NEMATIC CRYSTAL WITHOUT FERROFLUID. - Figures 2 and 4 give the slope of the optical axis of the material $(\operatorname{tg} \theta)$ at a given point $X(X=$ nor-

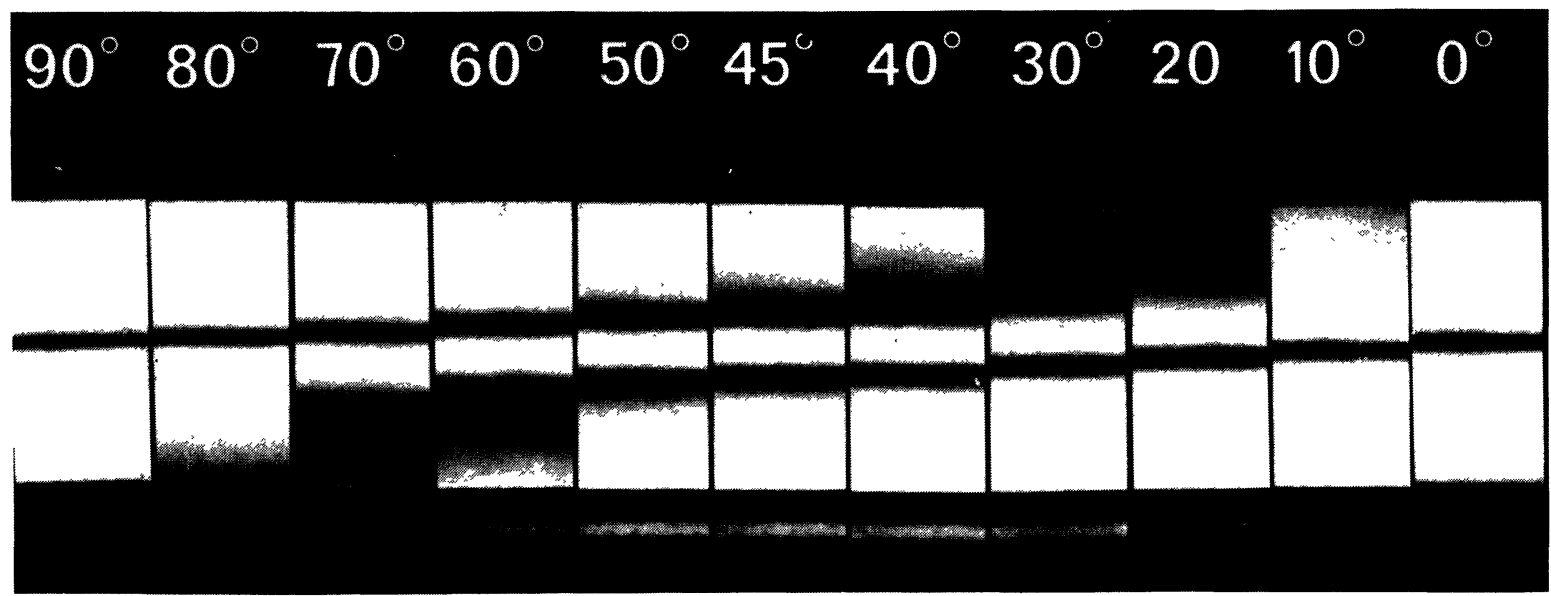

Plate I. - Successive aspects of the channel as viewed in polarized light for different $\theta$ angles between capillary axis and polarizers. 


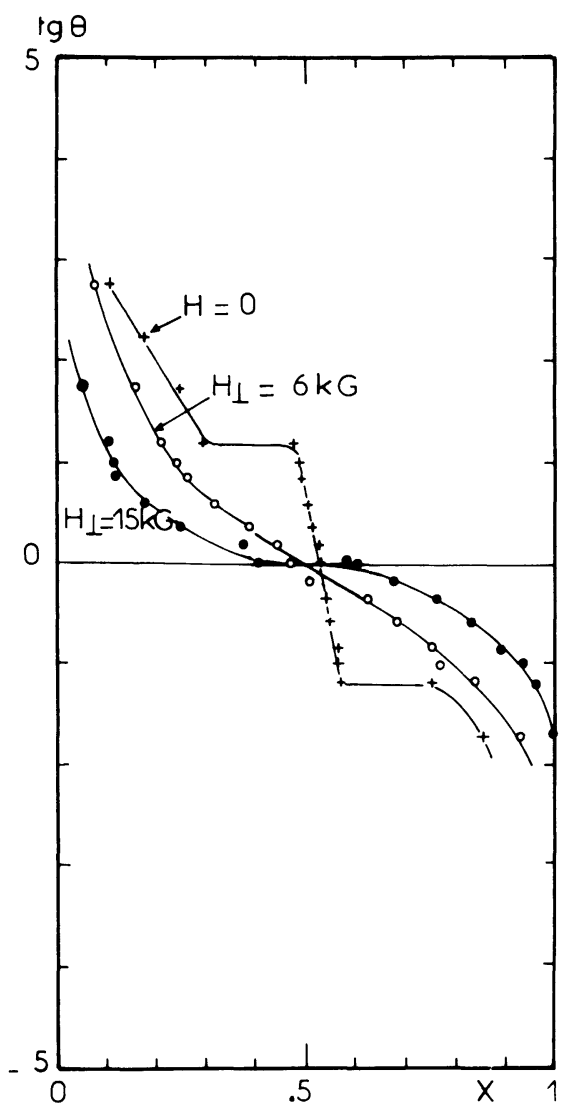

Fig. 2. - Slope of the optical axis across the channel of pure nematic lyomesophase for different values of the perpendicular magnetic field.

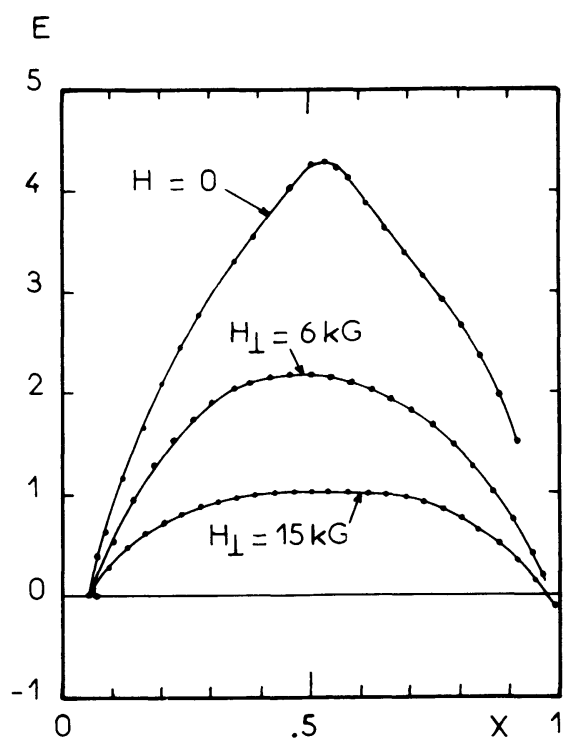

Fig. 3. - Orientation profiles of the nematic lyomesophase deduced from the data of figure 2 for different perpendicular magnetic fields.

malized position of the associated black line appearing in the field) for different values and orientations of the magnetic field.
We get the envelope $E$ of the disk orientation by integrating

$$
E=\int_{x_{0}}^{x} \operatorname{tg} \theta d x
$$

$x_{0}$, position of the most external visible line taken as reference.

The orientation profile could be represented equally well by $\theta$ or $\operatorname{tg} \theta$ versus $X$. The $E$ plot allows an easier visualization of the profile. The micelle disk is tangent to the curve $E(x)$ at any point $X$ across the channel. The shape of the curve also strongly suggests that the initial orientation profile (for $H=0$ ), reveals the velocity profile of the flow which has been established during the filling stage.

Figures 3 and 5 give the orientation profile deduced by the integration for the field values and orientations of figures 2 and 4 . It can be noted that a perpendicular magnetic field tends to flatten the profile whereas a parallel field acts in the opposite sense. This agrees with the experimental fact which has been independently established that the plane of the micelles orient parallel to the magnetic field, i.e. the central part between the two black lines for $\theta=\pi / 4$ is favoured in perpendicular field and the two black lines move apart. This gives us a criterion to decide between the

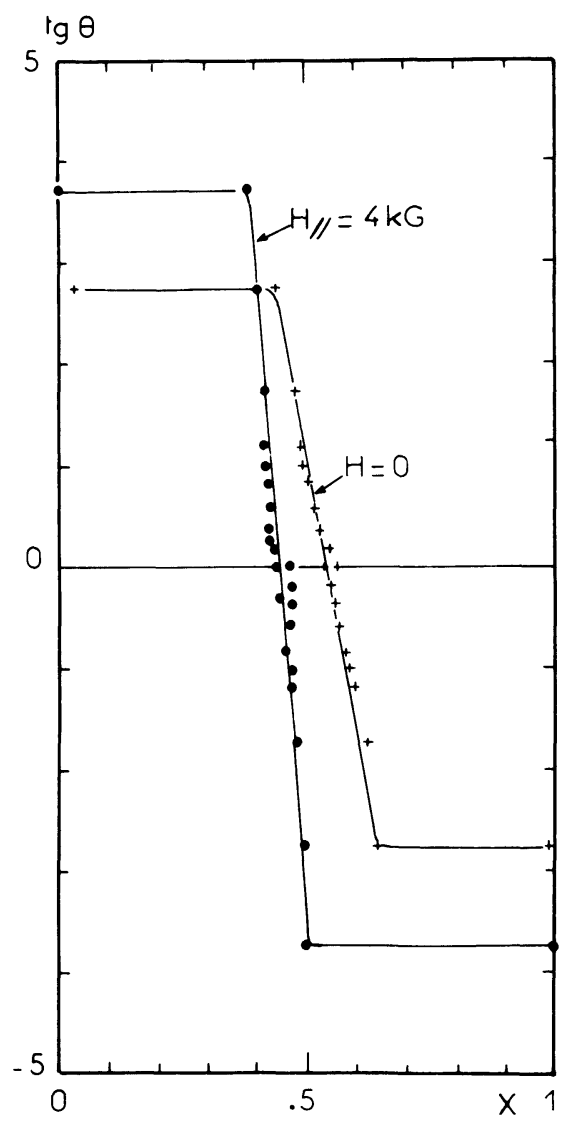

Fig. 4. - Slope of the optical axis across the channel of pure nematic lyomesophase for a parallel orientation of the magnetic field. 


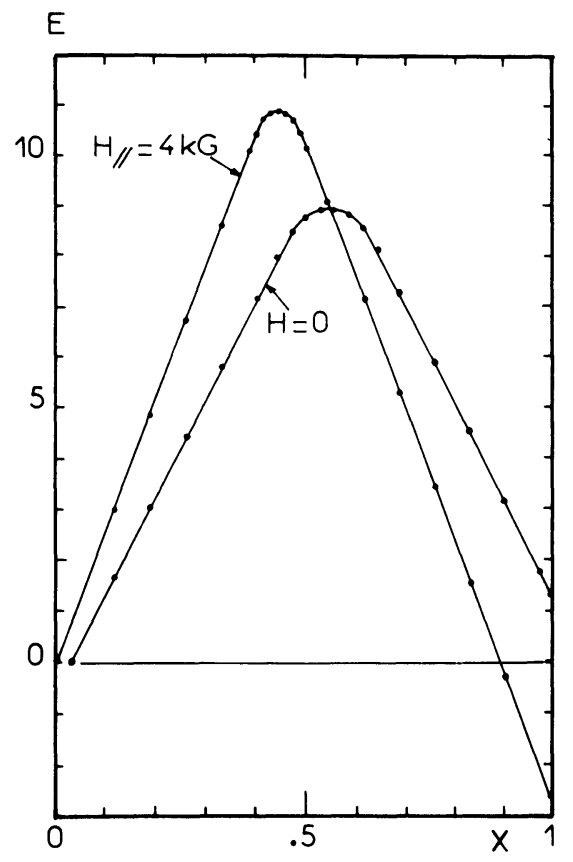

Fig. 5. - Orientation profiles deduced from the data of figure 4 for a parallel magnetic field.

two possibilities mentioned above in the determination of the profile.

Figures 6 and 7 show the time dependence effects. A static magnetic field $(6 \mathrm{kG})$ has been applied suddenly at $t=0$. The distance $\Delta l$ between the two central black lines increases slowly towards a final equilibrium value $\Delta l_{\mathrm{f}} . \Delta l_{\mathrm{f}}-\Delta l$ is plotted on a logarithmic scale versus $t$ in figure 6. Then, at time $t_{1}$, the field is shut off and $\Delta l$ relaxes towards a $\Delta l_{0}$ value. $\Delta l-\Delta l_{0}$ is plotted versus $t-t_{1}$ in figure 7 . The behaviour is approximately exponential with a typical time constant of several minutes. This fact clearly indicates the presence of a restoring force acting on the orientation of the micelles.

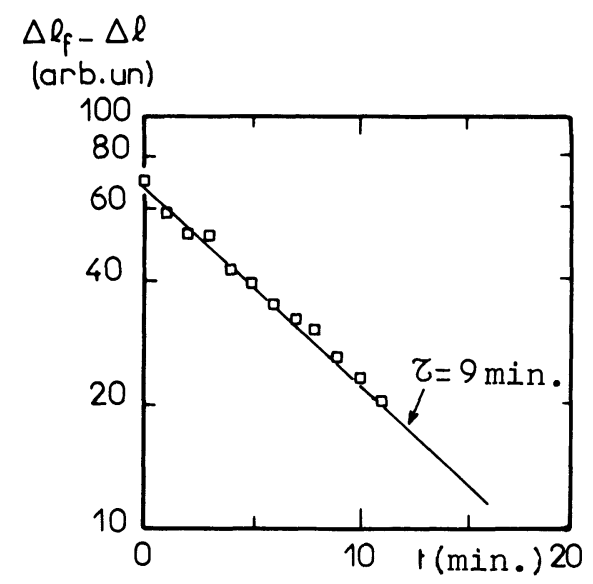

Fig. 6. - Time dependence effect on pure nematic lyomesophase : a static perpendicular field $(6 \mathrm{kG})$ is suddenly applied at $t=0$. The departure from equilibrium is plotted in logarithmic units versus $t$.

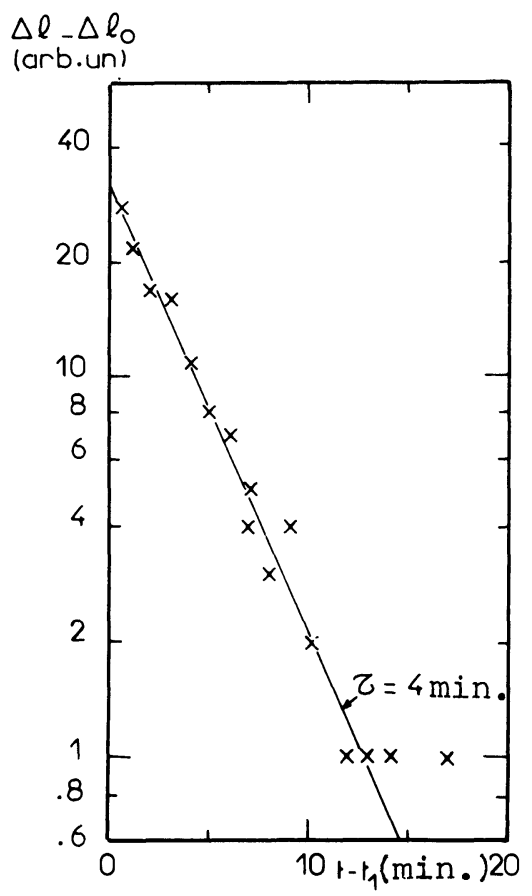

Fig. 7. - Time dependence effect : on pure magnetic lyomesophase : at time $t_{1}$, the magnetic field is suppressed and the relaxation is plotted in logarithmic units versus $t-t_{1}$.

3.2 TYPE II NEMATIC-LYOTROPIC CRYSTAL WITH FERROFLUID. - The same measurements have been performed with the type II-ferrofluid mixture. The slope of the optical axis for a parallel, a perpendicular or no field are plotted in figure 8, and the corresponding orientation profiles are shown in figure 9.

The time dependence is shown in figures 10 and 11 where the widening of the two central black lines is plotted logarithmically versus time. Figure 10 shows the effect of the application of a perpendicular field $(4.5 \mathrm{G})$ at time $t=0$. Figure 11 shows the relaxation after the suppression of a perpendicular magnetic field at time $t_{1}$. As in the case of pure nematic lyomesophase, an exponential law is approximately observed. It has to be noted, however, that for time periods longer than $10 \mathrm{~min}$, the behaviour departs noticeably from an exponential. This probably results from a progressive gliding of the orientation near the walls.

4. Discussion and conclusion. - Comparing the two sets of measurements, it is clear that the two behaviours are analogous except for a striking factor of $10^{3}-10^{4}$ in the magnetic fields necessary to impose a well defined orientation profile. This agrees with the mechanical coupling introduced by Brochard and de Gennes [6]; instead of coupling to the small magnetic anisotropy of the pure nematic lyomesophase of the order of

$$
V \chi_{\mathrm{a}} H_{1}^{2}
$$

where $V$ is the volume of a grain; $\chi_{\mathrm{a}}$ the magnetic 


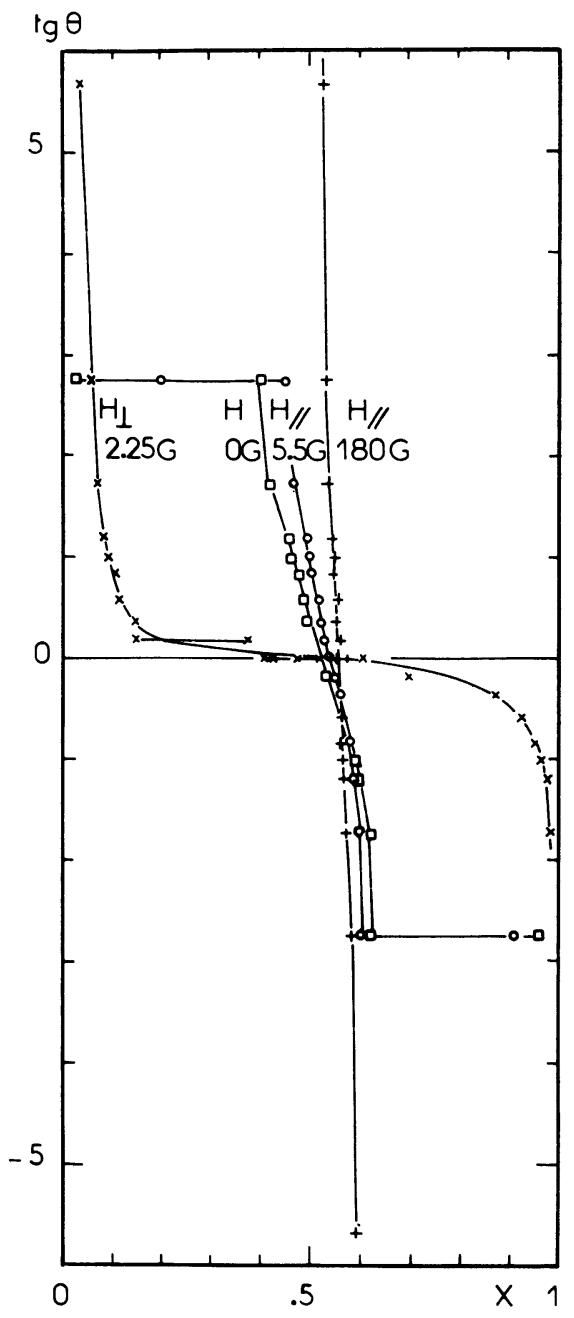

Fig. 8. - Slope of the optical axis across the channel of ferrofluidnematic lyomesophase for different values and orientation of the magnetic field.

anisotropy and $H_{1}$ the magnetic field applied in the pure type II case, one acts directly on the magnetic moment of the grains. The grains are then mechanically coupled with the surrounding micelles. The magnetic torque is of the order of :

$$
V M_{\mathrm{S}} \mathfrak{L}\left(\mathrm{H}_{2}\right) \mathrm{H}_{2}
$$

where $M_{\mathrm{S}}$ is the bulk magnetization at saturation of the magnetic material; $\mathcal{L}(H)$ is the Langevin function; $\mathrm{H}_{2}$ is the magnetic field applied in the type II-magnetic colloid case. Taking $\chi_{\mathrm{a}} \sim 10^{-7}, M_{\mathrm{S}} \sim 5 \times 10^{2}$, $H_{2}=5 \mathrm{G}, \mathfrak{L}\left(H_{2}\right) \sim 10^{-1}$, one gets $H_{1} / H_{2} \sim 10^{4}$.

This effect needs elongated grains to work. The grains of the ferrofluid used look roughly spherical, but as noted above, they align in chains which act as acicular objects. This is confirmed by optical and electron microscope observations.

The starting orientation profile is not very reproducible and changes slightly from one experiment to another. Certainly, it depends on uncontrolled boundary conditions on the walls and on the shear rate imposed during the filling. This lack of reproducibility

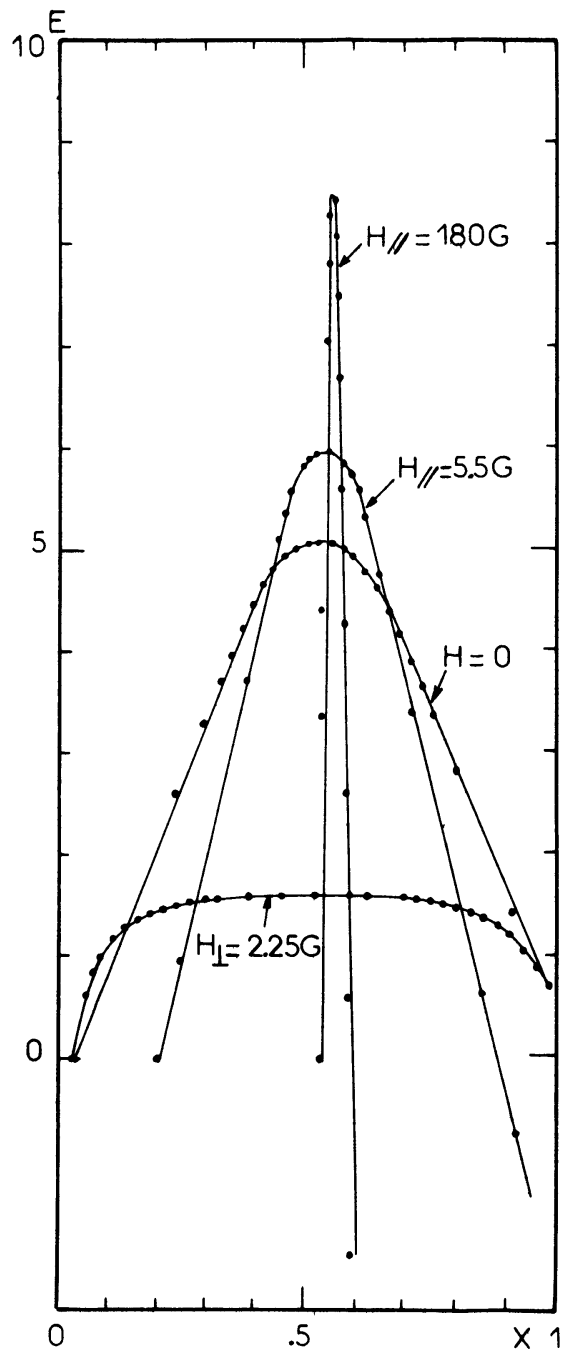

Fig. 9. - Orientation profiles deduced from the data of figure 8 for different values and orientation of the magnetic field. For the $180 \mathrm{G}$ curve, the $E$-scale has been expanded by a factor 40 .

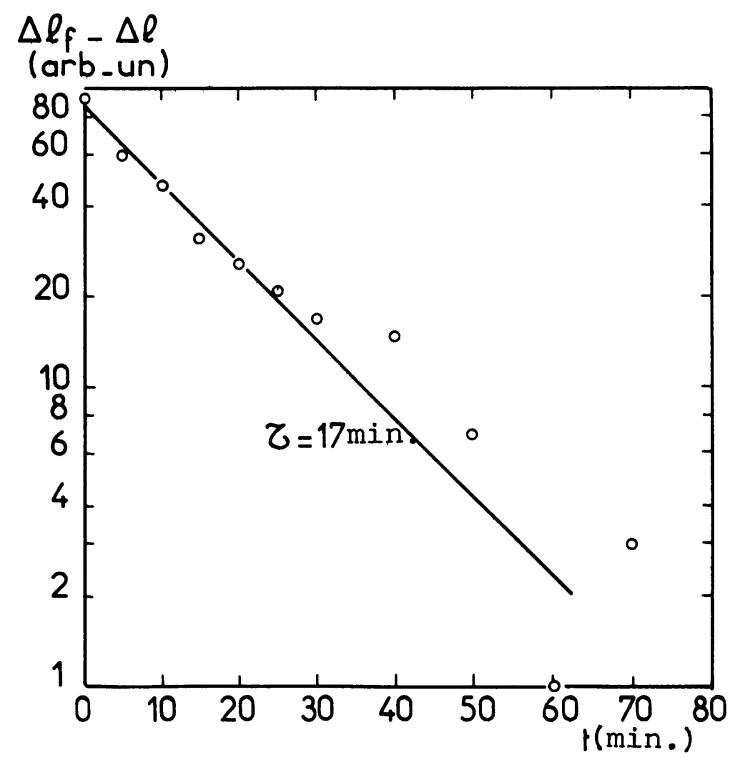

Fig. 10. - Time dependence effects on ferrofluid-nematic lyomesophase : exponential melting up of the equilibrium orientation after a perpendicular static field $(4.5 \mathrm{G})$ has been applied at $t=0$. 


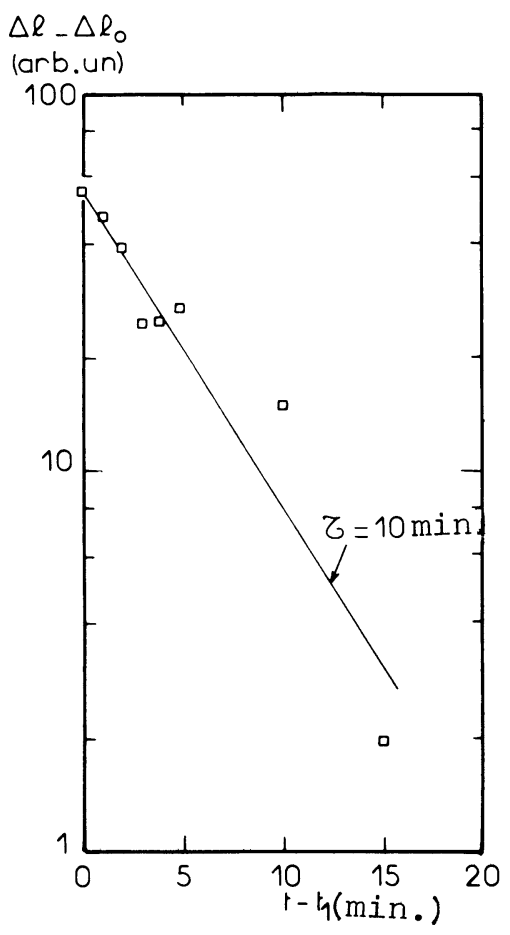

impedes further progress towards a quantitative description.

This coupled type-II ferrofluid [11] is the first stable ferronematic reported in the literature. Earlier attempts dealt with thermotropic liquid crystals. The main problem in thermotropics is that the nematic order is not satisfied near the grains (which are coated with a surfactant inducing strong deformations in the nematic) and the grains are rejected. Here, the system is flexible enough to accept magnetic grains.

Acknowledgments. - We are grateful to L. Strzelecki for the synthesis of very pure sodium decyl sulphate, to Y. Galerne for stimulating discussions and J. Charvolin for his interest in nematic lyomesophases.

Fig. 11. - Time dependence effects on ferrofluid-nematic lyomesophase : exponential relaxation of the orientation when the perturbing perpendicular field is suppressed at time $t_{1}$.

\section{References}

[1] Lawson, K. D. and Flautt, T. J., J. Am. Chem. Soc. 89 (1967) 5490.

[2] Rosevear, F. B., J. Soc. Cosmet. Chem. 19 (1968) 581.

[3] Radley, K., Reeves, L. W. and Tracey, A. S., J. Phys. Chem. 80 (1976) 174

[4] Radley, K. and Saupe, A., Mol. Cryst. Liq. Cryst. 44 (1978) 227.

[5] Funiwara, F. Y. and Reeves, L. W., Can. J. Chem. 56 (1978) 2178.

[6] Brochard, F. and de Gennes, P. G., J. Physique 31 (1970) 691.
[7] Charvolin, J., private communication.

[8] Chandesris, D., Martinet, A. and Strzelecki, L., Revue Phys. Appl. 12 (1977) 873.

[9] Peterson, E. A. and Krueger, D. A., Proc. Intern. Adv. Course \& Workshop "Thermomechanics Magn. Liq." (Hemisphere Publ. Corp. Washington) 1978.

[10] Anthore, R., Petipas, C., Chandesris, D. and Martinet, A., J. Physique Colloq. 38 (1977) C2-203.

[11] Patent number : 7825 744, Sept. 7, 1978. 